\title{
Using perspective guidance overlay to improve UAV manual control performance
}

\author{
Jochum Tadema $^{* a}$, Eric Theunissen ${ }^{\mathrm{a}, \mathrm{b}}$, Joris Koeners ${ }^{\mathrm{b}}$ \\ ${ }^{a}$ Netherlands Defence Academy, Het Nieuwe Diep 8, 1781 AC, Den Helder, The Netherlands; \\ ${ }^{\mathrm{b}}$ Delft University of Technology, Mekelweg 4, 2628 CD, Delft, The Netherlands
}

\begin{abstract}
The guidance information that is available to the UAV operator typically suffers from limitations of data update rate and system latency. Even when using a flight director command display, the manual control task is considerably more difficult compared to piloting a manned aircraft. Results from earlier research into perspective guidance displays show that these displays provide performance benefits and suggest a reduction of the negative effects of system latency. The current study has shown that in case of limitations of data update rate and system latency the use of a conformal sensor overlay showing a perspective presentation of the trajectory constraints is consistently superior to the flight director command display. The superiority becomes more pronounced with an increase in data latency and a decrease in update rate. The fact that the perspective pathway overlay as used in this study can be implemented on any graphics system that is capable of rendering a set of 2-D vectors makes it a viable candidate for upgrades to current systems.
\end{abstract}

Keywords: UAV, manual control, perspective guidance overlay, latency, update rate

\section{INTRODUCTION}

For the manual control of the flight path of Unmanned Aerial Vehicles (UAVs), the instruments provided in a UAV Control Station (UCS) are similar to those found in the cockpit of an aircraft. Typically the out-of-the-window view is represented by a forward looking camera and a plan-view display containing an electronic map is used for navigational conformance monitoring. Obviously, the richness of the visual-, motion- and auditory cues that is available to the pilot of a manned aircraft is not available to the UAV operator. In addition, the information that is available to the UAV operator typically suffers from low update rates and resolution, caused by sensor and data link bandwidth limitations. Furthermore, compared to manned aircraft, the data presented by the instruments in the UCS is subjected to an increased latency due to the (digital) data link. Even when using a flight director (FD) command display, the manual control task is considerably more difficult compared to piloting a manned aircraft. This paper explores the potential of an alternative guidance display concept because it is expected that it will allow better tracking performance with reduced control activity.

\section{BACKGROUND}

In 1978 NASA conducted a research program on developing landing techniques with which a pilot could consistently and safely land a remotely piloted research vehicle ${ }^{3}$. This study concluded that making remote landings from steep approaches using television required extremely high pilot workload. Operator experiences with current generation UAV systems also indicate that the manual landing task is extremely difficult $t^{6,7}$. Consequently, available information on United States Air Force class A aerospace mishaps indicates skill-based human error in manual control as a contributing factor in several mishaps in UAV operations ${ }^{10}$. Reported problems comprise difficulties in controlling aircraft glide path, sink rate, aim point, and airspeed on short final, as well as difficulties to correct unstable short final approaches. A reduction in these control related accidents might be achieved through the improvement of path tracking performance.

*j.tadema@nlda.nl; phone: +31-223-657545; fax: +31-223-657319; http://www.uav.nl

Enhanced and Synthetic Vision 2007, edited by Jacques G. Verly, Jeff J. Guell, Proc. of SPIE Vol. 6559, 65590C, (2007) · 0277-786X/07/\$18 · doi: 10.1117/12.719238 
In the past twenty-five years, several studies have demonstrated that a well designed perspective guidance display format allows pilots to achieve a better path tracking performance with lower control activity than an FD command display. In general, the differences in performance between the two concepts become more pronounced with an increase in trajectory complexity. Hence, one might expect that for UAV manual control, the presentation of the future trajectory provided by a perspective guidance display can contribute to increased path tracking performance.

However, there is a second reason to consider the use of an integrated perspective display. In Filarsky and Hoover ${ }^{2}$, a socalled highway-in-the-sky (perspective) display format was compared to a conventional FD format. Both systems suffered from the same system latency, but pilot comments suggested that the effects were not so noticeable with the perspective display format. This suggests that in case of system latency, pilots may perform better using a perspective presentation of the future trajectory than an FD. If this is indeed the case, this finding is of particular importance for the manual control of UAVs.

The generation of a basic perspective pathway overlay can be performed without any dedicated 3-D graphics hardware and is well within the capabilities of today's UCSs. The goal of the present study is therefore to investigate whether the finding regarding the reduced prominence of control loop latency resulting from perspective display concepts can be replicated for a high precision path tracking task for a system with vehicle dynamics typical for a UAV over a range of data latencies and update rates.

\section{EXPERIMENTAL DESIGN}

To investigate whether the finding regarding the reduced prominence of control loop latency resulting from perspective display concepts can be replicated for a high precision path tracking task for a system with vehicle dynamics typical for a UAV over a range of data latencies and update rates, an experiment has been conducted. In this experiment a baseline representative for the current state-of-the-art UCS instrumentation is compared with a concept that includes conformally integrated trajectory preview into the sensor video and filtering of down-linked position data. This section describes the method followed for this evaluation.

\subsection{Simulation environment}

The UCS simulation environment shown in Figure 1 is located at the Royal Netherlands Naval College (RNLNC) in Den Helder. It has been developed during our research in this field ${ }^{4}$. Previously, it has been used for UAV mission management research on intermediate- and outer-loop control, i.e., operator interaction with the autopilot in terms of heading, speed and altitude adjustments and the repositioning of waypoints.

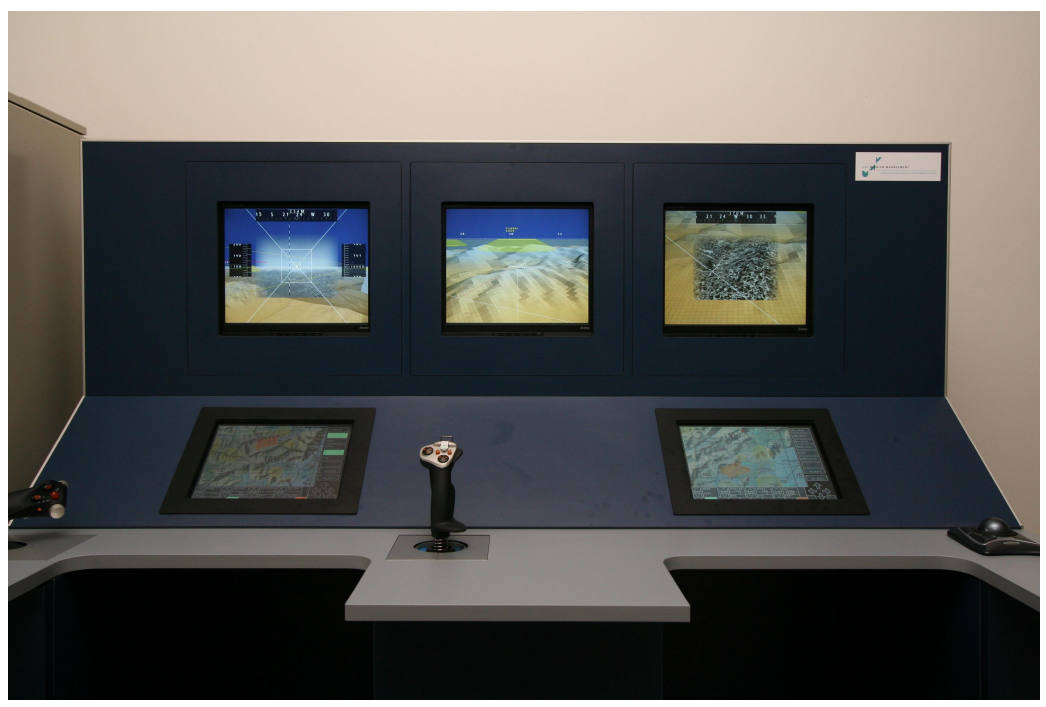

Figure 1. UCS simulation environment at the RNLNC

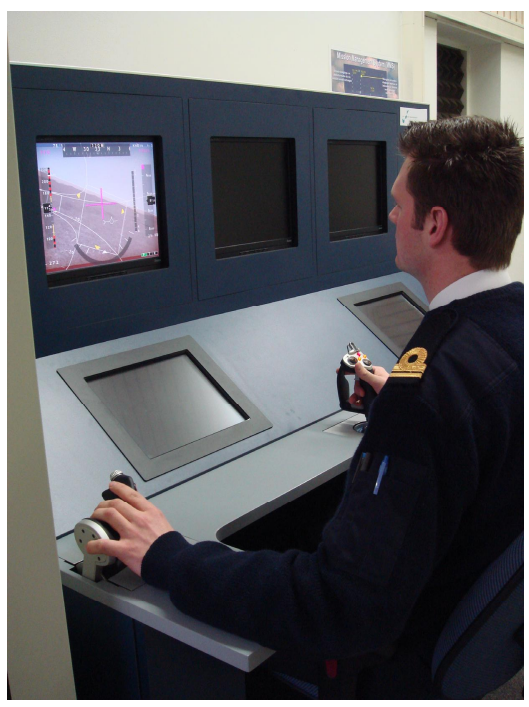

Figure 2. Manual control set-up 
Due to the nature of the connection between UCS and UAV, time delay of control signals (operator input) and feedback signals (telemetry information and video imagery) exist. Additionally limitations in available data link bandwidth and sensor characteristics impose restrictions to signal sample rate and sample size, i.e., quantizing resolution and quantizing range (e.g. field of view of imaging sensors). These limiting factors cause temporal and spatial uncertainty in the system state that is presented to the operator and thus hamper the ability of identifying and solving potential problems or conflicts, especially when performing higher bandwidth (inner-loop) control. To support the inner-loop control study presented in this paper, the UCS simulator was extended with the capability to systematically vary sensor and data link properties and the possibility to use data-filtering on incoming position data ${ }^{5}$. Figure 3 provides an overview of the required components.

During the experiment, participants used the head-level display and stick and throttle on the left side of the simulator (Figure 2). The head-level display presented simulated video imagery from the UAV's fixed, nose-mounted camera, along with head-up display (HUD) guidance symbology. The simulated imagery was created from a realistic database model of the area surrounding the Reno/Tahoe International Airport, Nevada.

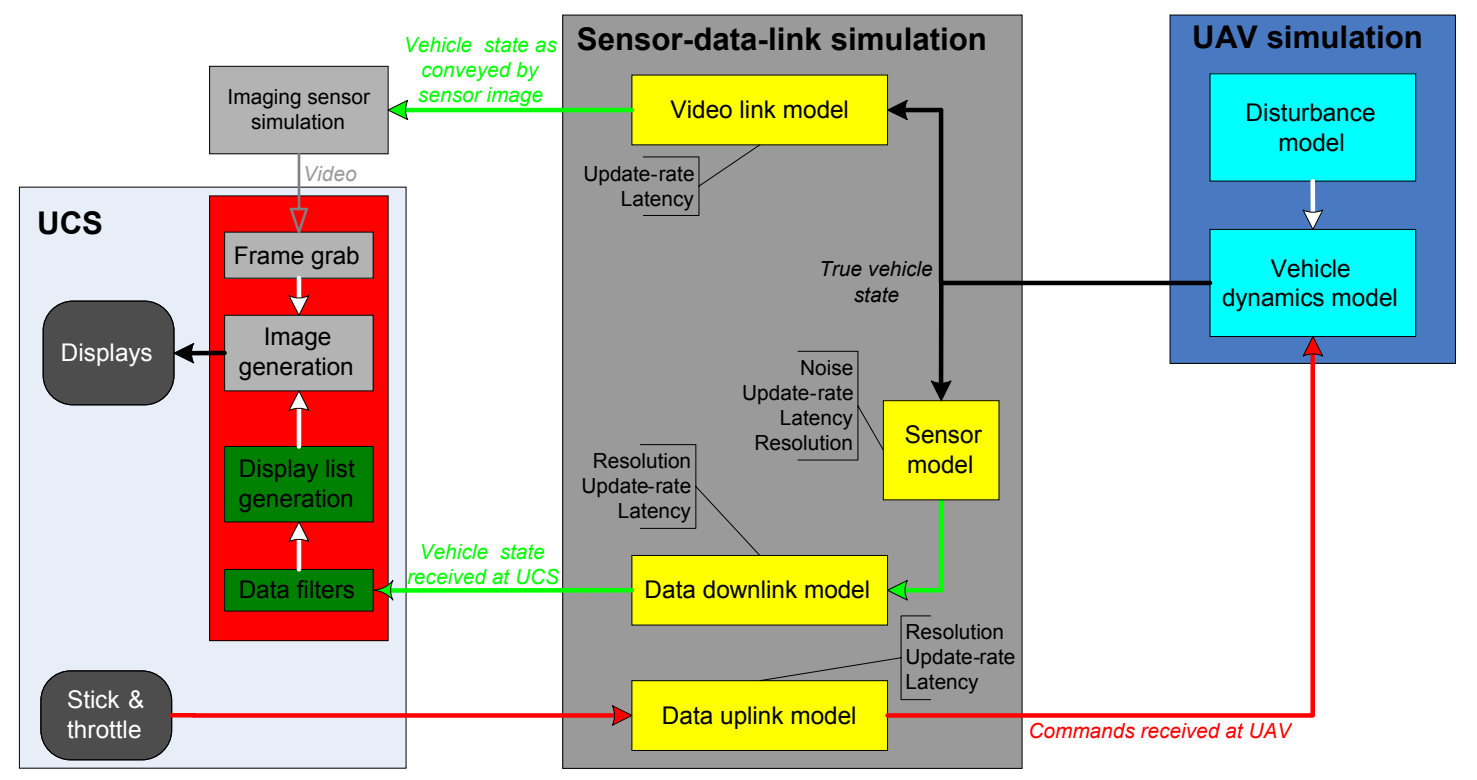

Figure 3. Overview of the required components in the basic simulation environment ${ }^{5}$

\subsection{Independent variables}

The study addresses three independent variables:

- HUD instrument concept;

- control loop delay, comprising of data uplink delay, data downlink delay and video link delay (Figure 3);

- position data update rate.

The different UAV HUD configurations implemented in the UCS simulator are shown in figures 4-7. Figures 4 and 6 represent the baseline situation $I_{l}$, in which the video imagery is augmented with conventional HUD elements providing information on the aircraft's heading, speed, altitude and attitude, as well as a flight path marker to represent the direction of the velocity vector. FD command bars provide the ('follow-the-needle') guidance cues along the reference flight path. Figures 5 and 7 represent the HUD configuration $I_{2}$, in which conformally integrated trajectory preview and extrapolation of low update rate position data were implemented ${ }^{8}$. As can be observed from these figures, the perspective pathway is rendered by means of a wireframe representation. All required transformations, the perspective projection and 
the clipping are performed in software. Consequently, the overlay concept can be implemented on any graphics system that is capable of rendering 2-D vectors.

In both conditions, the simulated video imagery represents a fixed, nose mounted camera. Field of view, location and direction of the camera were not varied throughout the experiment.

The delay values chosen for this study (Table 2) follow from an analysis of the different sources of time delay, in which the total roundtrip signal delay for UAV control is estimated to be between $100-1100 \mathrm{~ms}$ for line of sight (LOS) connections ${ }^{9}$. The minimal value of this estimate represents a symmetrical link in which both up- and downlink contribute about $50 \mathrm{~ms}$ of delay, caused mainly by transceiver electronics. The maximum value includes encryption, error correction and the presence of a Ground Data Terminal (GDT), as well as an additional $375 \mathrm{~ms}$ delay in the video channel due to compression and decompression of the digital video.

The chosen parameter settings for the independent variables are given in Table 1-3. The resulting condition matrix is given in Table 4.

Table 1: HUD instrument conditions

\begin{tabular}{|c|lc|}
\hline HUD instrument & Flight instrumentation description & \\
\hline $\boldsymbol{I}_{\boldsymbol{1}}$ & Flight Director command bars, unfiltered position data & (Fig. 4,6) \\
\hline $\boldsymbol{I}_{\mathbf{2}}$ & Perspective trajectory preview + predictor, filtered position data & (Fig. 5,7) \\
\hline
\end{tabular}

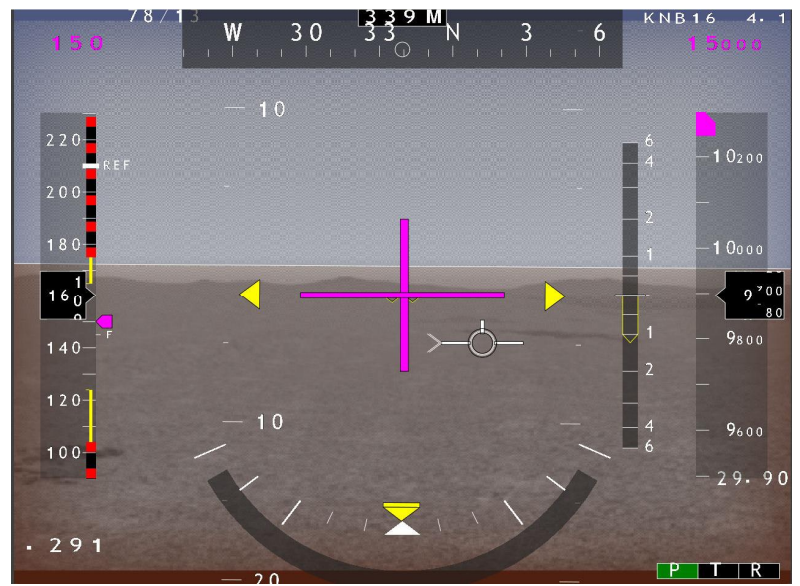

Figure 4. HUD instrument condition $I_{l}$ (en-route)

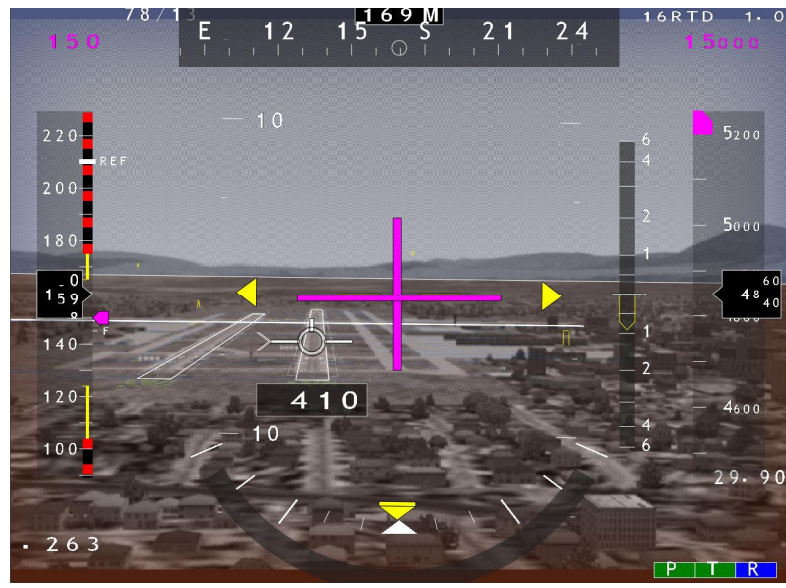

Figure 6. HUD instrument condition $I_{l}$ (short final)

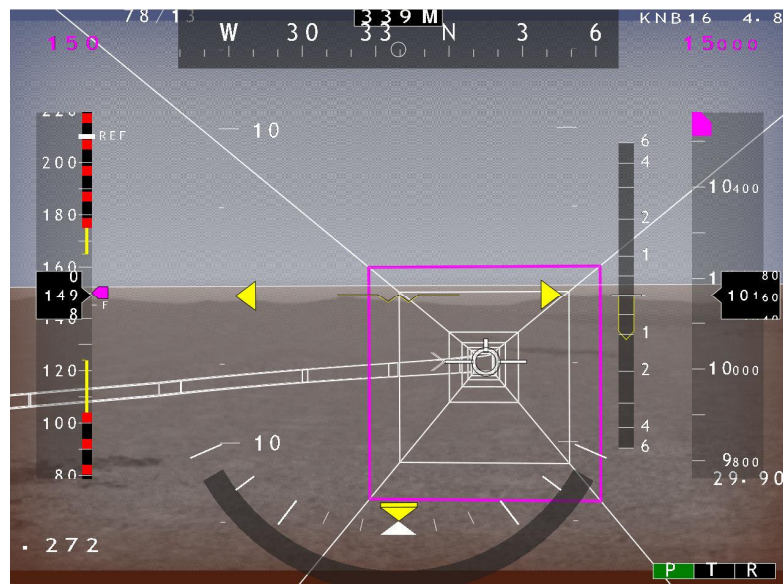

Figure 5. HUD instrument condition $I_{2}$ (en-route)

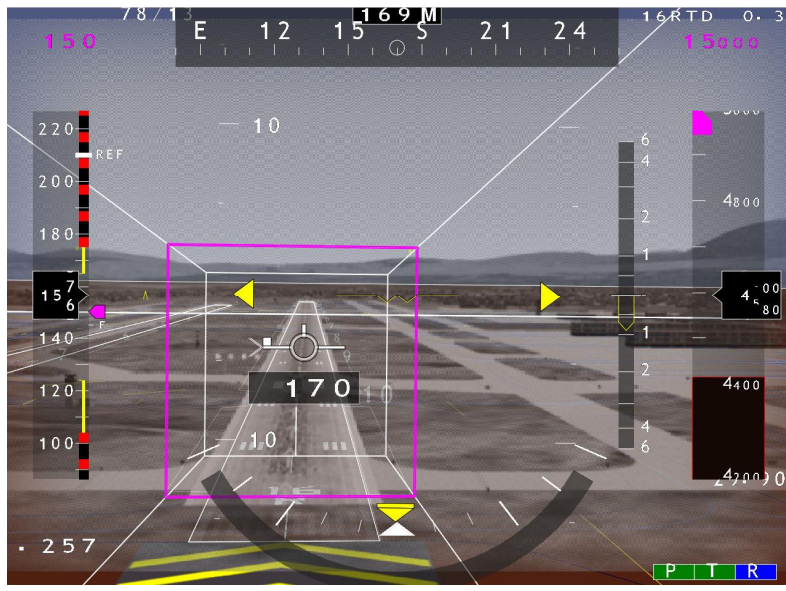

Figure 7. HUD instrument condition $I_{2}$ (short final) 
Table 2: Delay conditions

\begin{tabular}{|c|c|c|c|}
\hline Delay & $\tau_{\text {up }}[\mathrm{ms}]$ & $\tau_{\text {down }}[\mathrm{ms}]$ & $\tau_{\text {video }}[\mathrm{ms}]$ \\
\hline $\boldsymbol{\tau}_{\boldsymbol{1}}$ & 50 & 50 & 50 \\
\hline $\boldsymbol{\tau}_{\boldsymbol{2}}$ & 375 & 375 & 750 \\
\hline
\end{tabular}

Table 3: Position update rate conditions

\begin{tabular}{|c|c|}
\hline Sample period & $T_{\text {position }}[\mathrm{ms}]$ \\
\hline $\boldsymbol{T}_{\boldsymbol{1}}$ & 100 \\
\hline $\boldsymbol{T}_{\boldsymbol{2}}$ & 999 \\
\hline
\end{tabular}

Table 4: Condition matrix

\begin{tabular}{|c|c|c|c|}
\hline$\tau_{1} T_{1} I_{1}$ & $\tau_{1} T_{2} I_{1}$ & $\tau_{2} T_{1} I_{1}$ & $\tau_{2} T_{2} I_{1}$ \\
\hline$\tau_{1} T_{1} I_{2}$ & $\tau_{1} T_{2} I_{2}$ & $\tau_{2} T_{1} I_{2}$ & $\tau_{2} T_{2} I_{2}$ \\
\hline
\end{tabular}

\subsection{Task and dependent variables}

The task of the pilot was to track a complex, continuous descending approach trajectory and enter the runway as required for safe and smooth landing. Desired speed was $150 \mathrm{kts}$ and constant throughout the flight. The approach was flown in conditions of moderate wind and turbulence to increase realism and to demand continuous pilot control input. A single flight lasted about 5 minutes.

Task performance and associated workload were rated using the following measures:

- pilot rated handling qualities using the Cooper-Harper Rating (CHR) system ${ }^{1}$;

- RMS tracking errors;

- position of the vehicle and the direction of its velocity when crossing the runway threshold;

- $\quad$ RMS stick deflections, i.e., control effort.

Figures 8 and 9 depict the approach trajectory that was used in the evaluation.

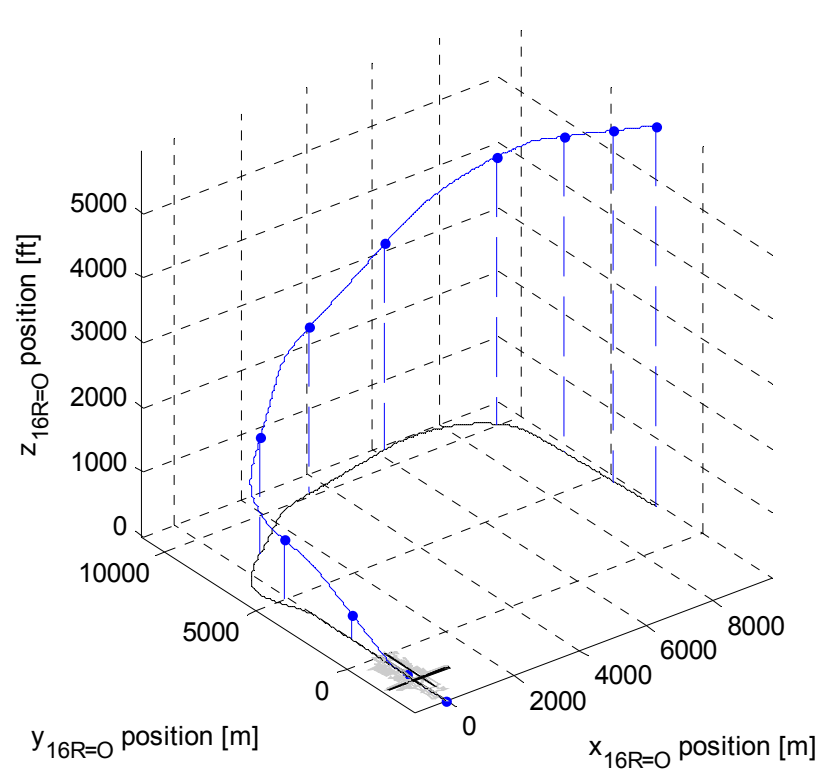

Figure 8. Commanded approach

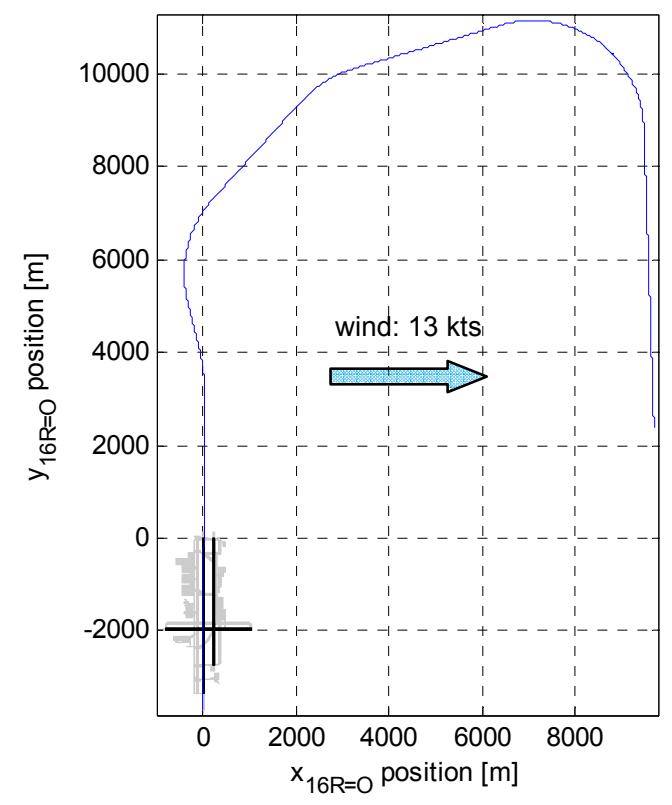

Figure 9. Ground track commanded approach 


\subsection{Procedure}

A total of 6 naval helicopter pilots from the Royal Netherlands Navy search and rescue squadron VGSQ 7 participated in the experiment. Participating pilots were first briefed on the task, the distinctive elements of the two display concepts and the CHR scale. Subsequently, flight preparation involved practice in the simulator to establish pilot confidence with the execution of the task for both display concepts and different data link settings. During these practice trials participants were informed on the data link parameter settings and encouraged to try out different control strategies to contribute to their comprehension of the control dynamics. On average, each participant needed roughly 30-45 minutes of practice time, performing 5 to 6 practice trials.

In the registered data trials, the participants flew each of the eight conditions given in Table 4 at least once. The decision to perform a second trial was based on the results of the first trial and available time. On average each participant performed 12 data trials. During the registered data trials participants were not informed on the data link settings, to not influence their opinion and comments.

Pilot comments were recorded on a questionnaire immediately following each trial. The completion of the questionnaire required a pilot rating using the CHR system and allowed for general comments about the experienced "feel of the system".

\section{RESULTS AND DISCUSSION}

This section discusses both the subjective pilot rated handling qualities and the objective measures retrieved from the registered data. Tracking performance was determined by the position errors relative to the commanded approach path. Whether or not the approach was successful, was judged on the state of the aircraft (position and direction of the velocity vector) in the vertical plane located at the runway threshold. Control effort was determined by an analysis of the pilot's control input actions.

\subsection{Pilot opinion}

Figure 10 summarizes the pilot CHR for each condition. Especially for the higher latency conditions considerable dispersion exists between pilot opinions on the evaluated conditions. For instance, pilot ratings for condition $\tau_{2} T_{1} I_{I}$ range from fair level 1 (CHR 3) handling qualities to uncontrollable (CHR 10). Remarkably, in the cases described by pilots as being uncontrollable, control was not lost at any point of the approach and performance even met the requirements for a successful approach.

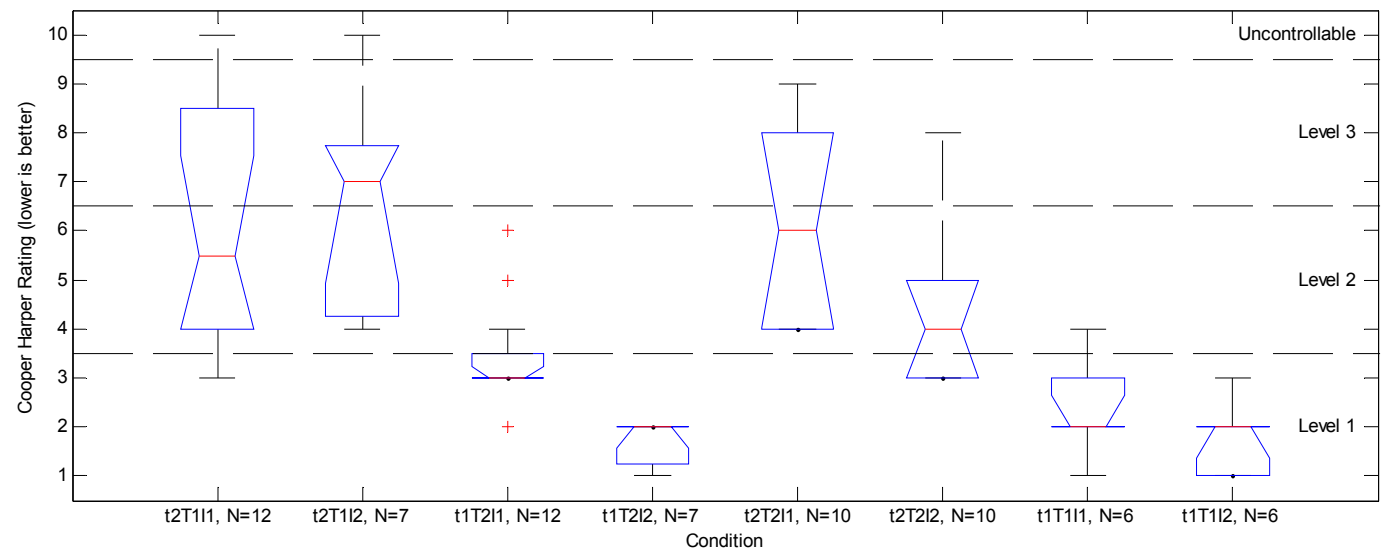

Figure 10. Pilot rated handling qualities 
Nevertheless, on average the data does indicate the following:

- increasing the position sampling period from $T_{1}$ to $T_{2}$ results in less distinct handling qualities degradation than increasing the latency condition from $\tau_{1}$ to $\tau_{2}$;

- link latency condition $\tau_{2}$ results in a reduction of the handling qualities from level 1 to level 2 or 3 for both instrument conditions;

- for link conditions $\tau_{1} T_{1}, \tau_{1} T_{2}$ and $\tau_{2} T_{2}$, pilot opinions state that instrument condition $I_{2}$ provides improved handling qualities over $I_{l}$.

Taking into account the dispersion in pilot ratings, instrument condition $I_{2}$ provides an improvement of the handling qualities for link condition $\tau_{2} T_{2}$ from a borderline level 2-3 $\left(I_{1}\right)$, towards a borderline level 1-2. For link condition $\tau_{2} T_{1}$ pilot ratings suggest borderline level 2-3 performance for both instrument conditions.

Additional remarks from the pilots affirmed that the evaluated control loop delays generally cause overcompensation. If the pilots succeeded in keeping tracking errors small, the high latency, low update rate conditions could be kept controllable but these cases did possess noticeably less margin for error. Additionally, pilots indicated that deficiencies were more noticeable and disturbing on short final than during earlier parts of the approach. This was especially the case if the evaluated condition parameters resulted in considerable asynchrony between the synthetic guidance overlay and the video imagery, causing discrepancies in the cues used for control. In some cases, it was pointed out that the explicitly depicted aiming point of instrument condition $I_{l}$ was favorable in the final approach phase. Finally, pilots indicated that the perspective representation of the commanded flight trajectory contributed to the predictability and allowed anticipatory control strategies, causing less overcompensation and making the negative effects of the link delays less severe.

\subsection{Tracking performance}

Figures 11 and 12 summarize the RMS values of the cross track error (XTE) and the vertical track error (VTE) along the approach path. On average, this data indicates that:

- increasing the position sampling period from $T_{1}$ to $T_{2}$ results in less distinct tracking performance degradation than increasing the latency condition from $\tau_{1}$ to $\tau_{2}$;

- higher delays result in larger position errors and thus reduced tracking accuracy;

- the negative effects of low update rate position data seem more distinct for the control of the lateral motion;

- for all link conditions, instrument condition $I_{2}$ results in smaller lateral and longitudinal position errors than instrument condition $I_{l}$ during the approach phase, expressing more accurate tracking and thus higher tracking performance.

Additionally, for all link conditions, instrument condition $I_{2}$ results in a smaller dispersion of the position errors than instrument condition $I_{l}$ during the approach phase, expressing a more consistent tracking performance.

Finally, the data also suggests the possible performance gains resulting from instrument condition $I_{2}$ are larger when link conditions are worse. 


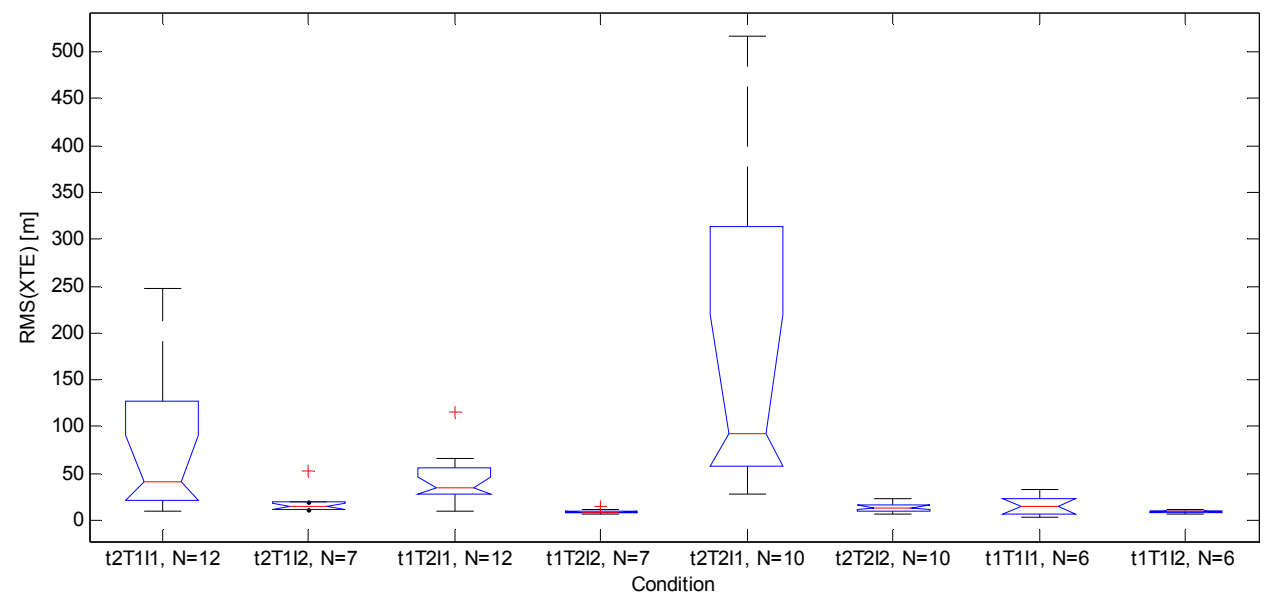

Figure 11. Tracking performance: RMS cross track errors during approach

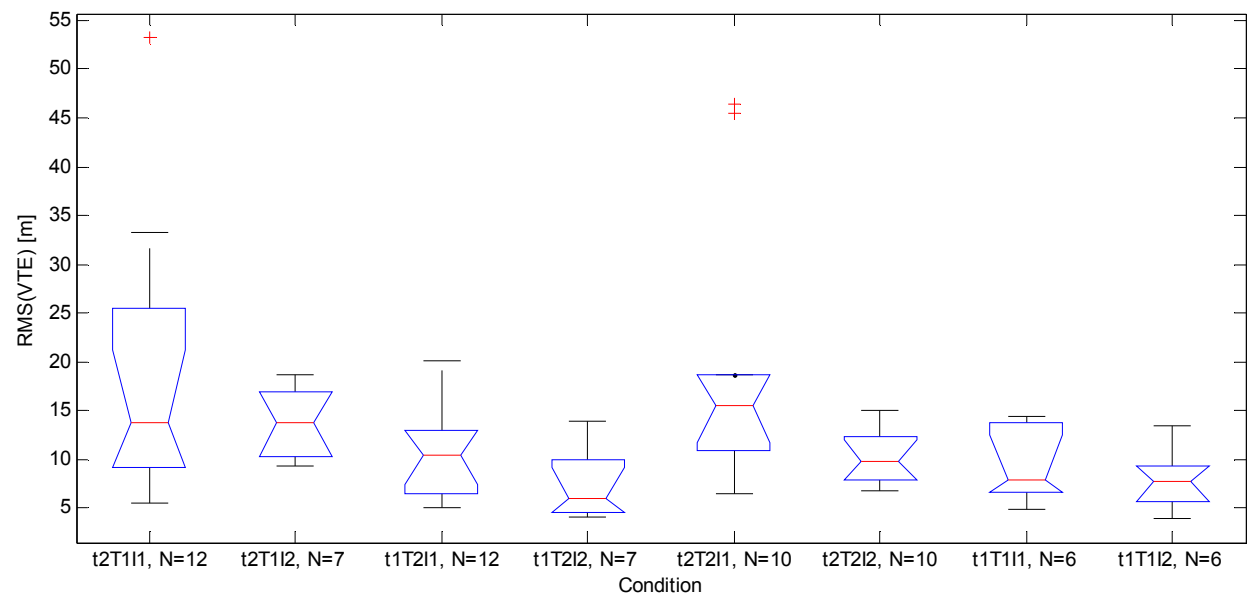

Figure 12. Tracking performance: RMS vertical track errors during approach

\subsection{Final approach performance}

An indication of the ability to successfully complete the landing is the position of the vehicle and the direction of its velocity when crossing the threshold. If the lateral position error at the threshold exceeds 15 meters or the track angle error (TAE) exceeds 5 degrees, the approach was rated as a missed approach. Figure 13 shows the ratio of missed approaches. This data, indicates that:

- missed approaches only occurred for delay condition $\tau_{2}$, suggesting an increase of the position sampling period from $T_{1}$ to $T_{2}$ results in less distinct final approach performance degradation than an increase of the latency from condition $\tau_{1}$ to $\tau_{2}$;

- the missed approach occurrence for instrument condition $I_{2}$ is smaller than that of instrument condition $I_{l}$, suggesting higher final approach performance for instrument condition $I_{2}$;

For the approaches considered successful, no evident trend for position errors at threshold could be observed (Figure 14). Comparing track angle errors (Figure 15), data indicates that for instrument condition $I_{2}$ vehicle direction was better aligned with the runway centerline, expressing increased final approach performance. 


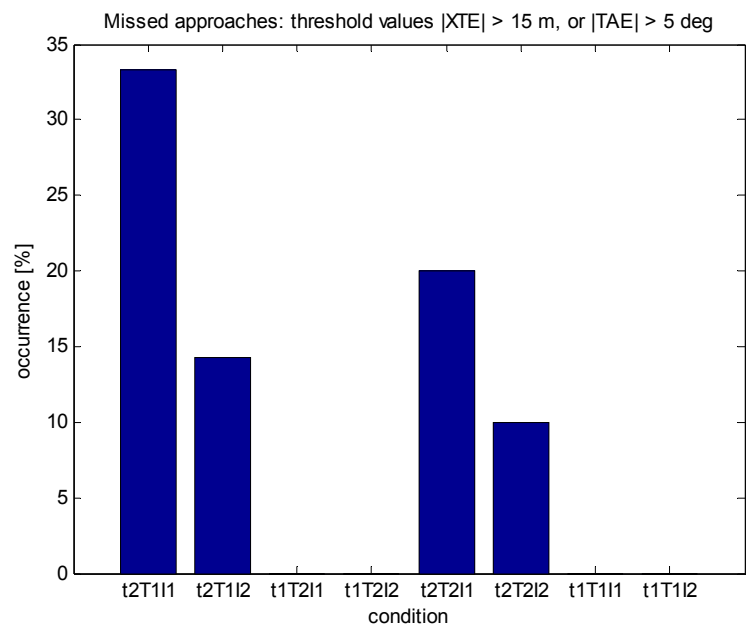

Figure 13. Final approach performance: missed approaches

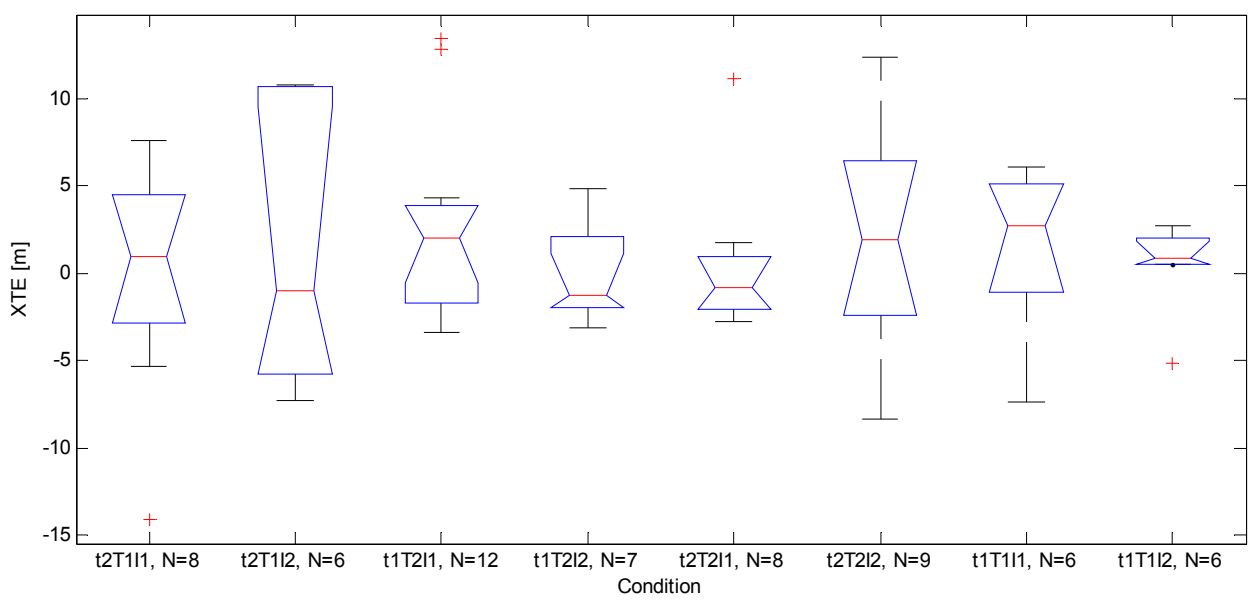

Figure 14. Final approach performance: cross track errors at runway threshold (missed approaches excluded)

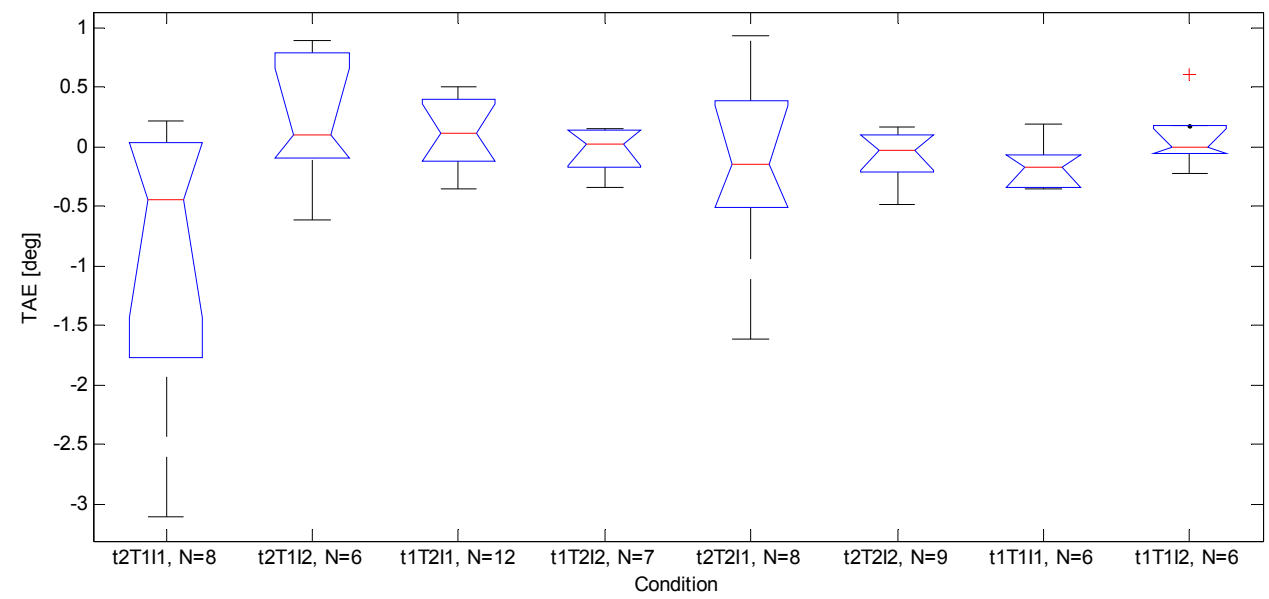

Figure 15. Final approach performance: track angle errors at runway threshold (missed approaches excluded) 


\subsection{Control effort}

Figures 16 and 17 present the data of roll and pitch control effort. The data, on average suggests that:

- increasing the position sampling period from $T_{1}$ to $T_{2}$ results in less distinct increase of control effort than increasing the latency condition from $\tau_{1}$ to $\tau_{2}$;

- the higher latency condition $\tau_{2}$ results in a higher level of applied control effort;

- instrument condition $I_{2}$ results in lower levels of applied roll control effort.

Finally, taking into account approach tracking performance, final approach performance and the corresponding control effort, data suggests the potential performance gains resulting from instrument condition $I_{2}$, are not accompanied by a considerable amount of increased control effort.

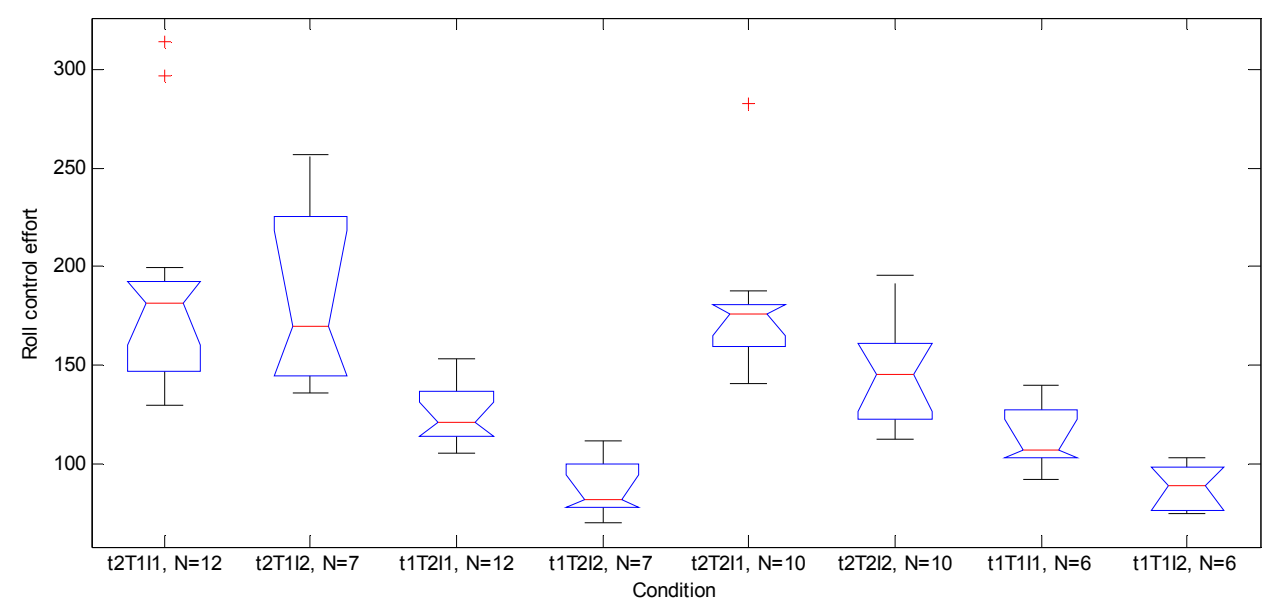

Figure 16. Roll control effort

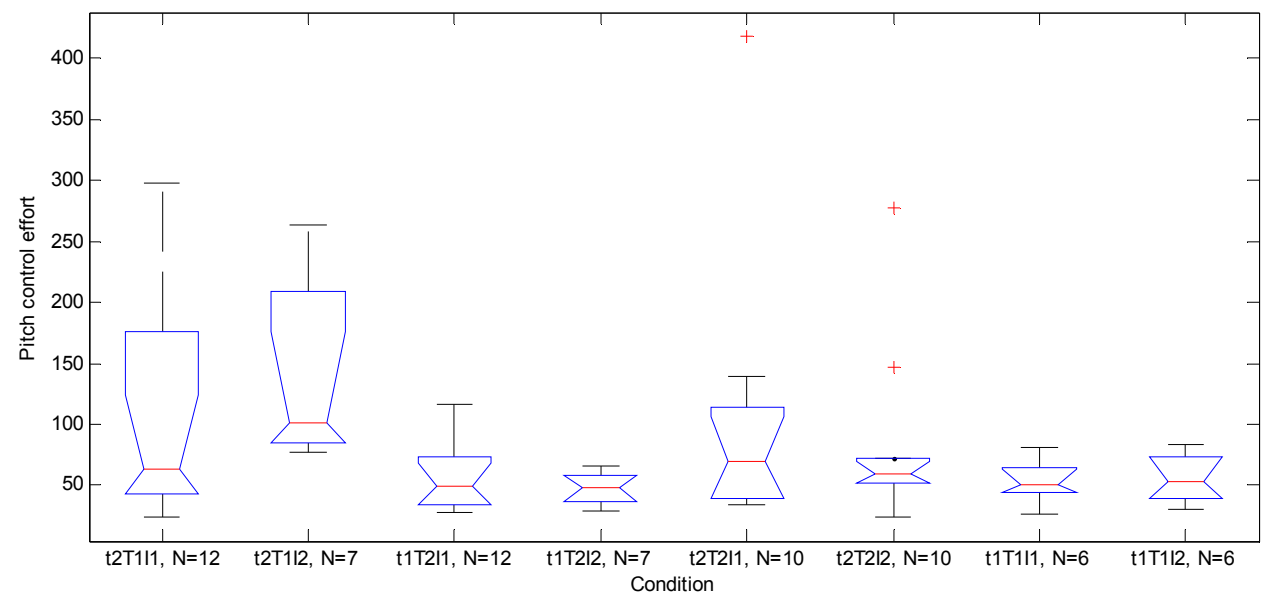

Figure 17. Pitch control effort 


\section{SUMMARY AND CONCLUSIONS}

Results from earlier research into perspective guidance displays show a performance benefit of these displays which becomes more pronounced when the complexity of the trajectory increases. The results from an early study into perspective guidance displays also suggest that as a side effect, a perspective display makes system latency less noticeable. The current study has shown that in case of limitations of data update rate and system latency the perspective guidance display is, in terms of precision tracking performance and required control effort, consistently superior to the FD command display. The superiority becomes more pronounced with an increase in data latency and a decrease in update rate. The fact that the perspective pathway overlay as used in this study can be implemented on any graphics system that is capable of rendering a set of 2-D vectors makes it a viable candidate for upgrades to current systems.

\section{ACKNOWLEDGEMENTS}

This study could not have been performed without the great support and enthusiasm of the participating pilots from the Royal Netherlands Navy search and rescue helicopter squadron VGSQ7, stationed at Naval Airbase De Kooy, Den Helder.

\section{REFERENCES}

1. G.E. Cooper and R.P. Harper Jr., The Use of Pilot Rating in the Evaluation of Aircraft Handling Qualities, NASA TN-D5153, 1969.

2. S.M. Filarsky and S.W. Hoover, The command flight path display, Naval Air Development Center, Warminster, Pennsylvania, 1983.

3. S.W. Gee and P.C. Carr, Development of Systems and Techniques for Landing an Aircraft Using Onboard Television, NASA Technical Paper 1171, Edwards, 1978.

4. A.A.H.E. Goossens, G.J.M. Koeners, J. Tadema and E. Theunissen, "Using Simulation to Refine UAV Operator Station Functional Requirements", Proceedings of the 2004 AIAA Modeling and Simulation Technologies Conference and Exhibit, Providence, Rhode Island, 2004.

5. G.J.M. Koeners, E. Theunissen, and J. Tadema, "Creating a Simulation Based Evaluation Environment for RPV Manual Control Concepts", Proceedings of AIAA Modeling and Simulation Technologies Conference and Exhibit, Keystone, 2006.

6. H.K. Pedersen, N.J. Cooke, H.L. Pringle and O. Connor, "UAV Human Factors: Operator Perspectives, Human Factors of Remotely Operated Vehicles", Advances in Human Performance and Cognitive Engineering Research, Volume 7, 21-33, Elsevier JAI Press, Oxford, 2006.

7. B.T. Schreiber, D.R. Lyon, E.L. Martin, H.A. Confer, Impact of prior flight experience on learning predator UAV operator skills, AFRL-HE-AZ-TR-2002-0026, Air Force Research Laboratory, Mesa, 2002.

8. E. Theunissen and R.E. de Bruijn, "Influence of position sensor properties when using a perspective flight path display", Proceedings of the 1999 AIAA Modeling and Simulation Technologies Conference, Portland Oregon, 1999.

9. S.C. de Vries, UAVs and control delays, TNO Defence, Security and Safety, TNO-DV3 2005 A054, Soesterberg, 2005.

10. United States Air Force Judge Advocate General's Corps Web site on Class A aerospace mishaps, http://usaf.aib.law.af.mil/ 